\title{
Effect of temperature on the structure formation in the magnetic fluid film subjected to perpendicular magnetic fields
}

\author{
S.Y. Yang ${ }^{\mathrm{a}, *}$, Y.F. Chen ${ }^{\mathrm{b}}$, Y.H. Ke ${ }^{\mathrm{c}}$, W.S. Tse ${ }^{\mathrm{a}}$, H.E. Horng ${ }^{\mathrm{c}}$, \\ Chin-Yih Hong ${ }^{\text {d }}$ H.C. Yang ${ }^{\mathrm{e}}$ \\ anstitute of Physics, Academia Sinica, Taipei 115, Taiwan \\ ${ }^{\mathrm{b}}$ Department of Physics, National Sun Yat-Sen University, Kaohsiung 804, Taiwan \\ ${ }^{\mathrm{c}}$ Department of Physics, National Taiwan Normal University, Taipei 116, Taiwan \\ ${ }^{\mathrm{d}}$ Department of Mechanical Engineering, Da-Yeh University, Chang-Hwa 515, Taiwan \\ ${ }^{\mathrm{e}}$ Department of Physics, National Taiwan University, Taipei 106, Taiwan
}

\begin{abstract}
The effect of temperature on the structure formation in the magnetic fluid film under perpendicular magnetic fields is studied. It was found that a higher field strength is required to make the magnetic particles agglomerated at a higher temperature. Also, the field strength corresponding to the formation of the ordered structure of magnetic particle columns in the film is increased when the temperature is increased. The results are due to the enhancement in the kinetic energy of magnetic particles at higher temperatures, therefore, allowing more magnetic particles to disperse into the liquid. To illustrate the relationship between the effect of temperature $T$ and of the magnetic field $H$ on the structure formation in the magnetic fluid film, a phase diagram is constructed for the structure pattern in the $H-T$ configuration. (C) 2002 Elsevier Science B.V. All rights reserved.
\end{abstract}

Keywords: Magnetic fluids; Structure; Temperature

\section{Introduction}

The studies on structural patterns of magnetic fluid films under external magnetic fields attract a great deal of interest. In 1970s, the agglomeration of magnetic particles in the magnetic fluid film under magnetic fields was observed [1]. Rosensweig and Popplewell then discussed the phase transition behavior, corresponding to the agglomeration of magnetic particles and of the magnetic fluid films under external magnetic fields [2]. Later, a series of structural patterns due to the phase separation [3-5] or the fingering of microdrops [6-8] in the magnetic fluid films under the influence of magnetic fields was reported. It was pointed out that the kinetic

*Corresponding author. Tel.: + 886-2-893-62-33; fax: +8862-293-09-439.

E-mail address: emesdolb@ms28.hinet.net (S.Y. Yang). energy of the magnetic particles and the external magnetic field are crucial to the agglomeration process.

In 1997, an ordered hexagonal structure formed by the magnetic columns was discovered by Hong et al. [5]. Also, a novel structure evolution in the highly homogeneous magnetic fluid film subjected to magnetic fields was observed [9]. It was further indicated that the ordered structure formation in the magnetic fluid film could be dominated by the magnetic field strength, the sweep rate of the field, the film thickness and the concentration of the fluid, etc. $[10,11]$. Since the agglomeration of columns is closely affected by the kinetic energy of the particles, the effect of temperature must also be influential to the ordered structure formation in the magnetic fluid film under magnetic fields. To clarify the role played by temperature in the formation of the ordered structure in the magnetic fluid film, the structure evolutions with magnetic fields are investigated at various temperatures here. 


\section{Experimental details}

The kerosene-based $\mathrm{MnFe}_{2} \mathrm{O}_{4}$ magnetic fluid of $5.6 \mathrm{emu} / \mathrm{g}$ was injected to a $6-\mu \mathrm{m}$-thick cell to form a magnetic fluid thin film. The film was posited inside a homemade temperature-controlling cell. The sample stage is constructed of copper and was connected to a water tank which serves to regulate the temperature via a constant circulation of water. To prevent heat convection, the sample space is vacuumed. A LakeShore Pt-111 temperature sensor, which is placed beside the film, is used to monitor the film temperature. The temperature resolution of the temperature-controlling cell is $0.1^{\circ} \mathrm{C}$. Next, the film and the cell were inserted into a pair of solenoids providing a magnetic field perpendicular to the film plane. The sweep rate of the magnetic field used here is $5 \mathrm{Oe} / \mathrm{s}$ and the maximum field strength available is $630 \mathrm{Oe}$. A microscope and a CCD camera were used to observe and record the structural pattern in the magnetic fluid film under magnetic fields.

\section{Results and discussion}

Under the zero field at a fixed temperature of $18.0^{\circ} \mathrm{C}$, the magnetic particles are monodispersed in the fluid. As the field strength $H$ exceeds a critical field $H_{0}$ $\left(=2.71 \mathrm{kA} / \mathrm{m}\right.$ for $\left.18.0^{\circ} \mathrm{C}\right)$, particles agglomerate to form magnetic columns located randomly in the film. When the field strength $H$ is further increased, more columns are formed. An ordered hexagonal structure formed by columns is achieved when $H$ reaches the critical field strength, $H_{\mathrm{h} 1}(=4.22 \mathrm{kA} / \mathrm{m})$. The ordered structure may be characterized by the average distance $d$ between two neighboring columns. When $H$ becomes higher than $H_{\mathrm{h} 1}$, new columns continue to appear, hence, $d$ is reduced. The variation in $d$ with the $H$ at various temperatures is shown in Fig. 1. Also, the images of a typical hexagonal ordered structure and its corresponding fast Fourier transformation are shown as the inset of Fig. 1. Since $d$ is changed while $H$ is increased, this state of the structure is referred to as a dynamic hexagonal structure.

If the field strength $H$ keeps increasing over $H_{\mathrm{p}}$ $(=6.13 \mathrm{kA} / \mathrm{m})$, the ordered structure remains unchanged until the $H$ exceeds a critical field $H_{\mathrm{s}}(=16.7 \mathrm{kA} / \mathrm{m})$. As a result, a plateau can be observed in the $d-H$ curve under $H_{\mathrm{p}}<H<H_{\mathrm{s}}$, as shown in Fig. 1. Contrast to the dynamic hexagonal structural state, $d$ of the current structure is independent of $H$. Hence, this state is defined as the first-level stable hexagonal structure. As $H$ is higher than $H_{\mathrm{s}}$, columns of the ordered structure begin to split into two until all the columns are split. The split of columns results in a decrease in $d$. Thus, it is also referred to as the dynamic hexagonal structure. Finally, the structure reaches a second-level stable hexagonal

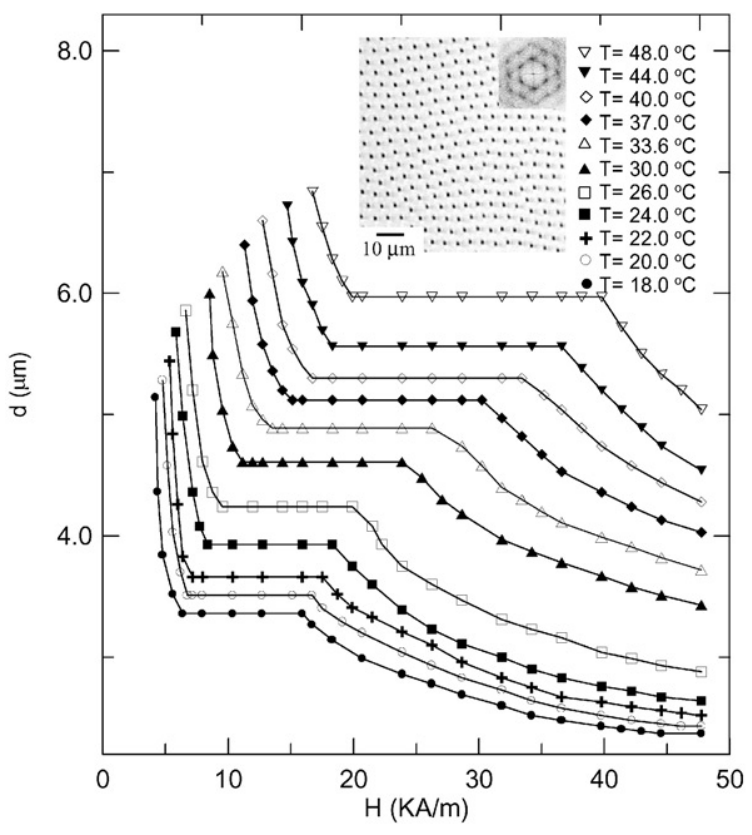

Fig. 1. Averaged distance $d$ between two neighboring columns of the ordered structure in the magnetic fluid film as functions of the applied magnetic field $H$ at various temperatures. The inset shows the typical image of the ordered structure and its corresponding fast Fourier transformation image.

structure when the $H$ is higher another critical field $H_{\mathrm{h} 2}$ $(=44.56 \mathrm{kA} / \mathrm{m})$.

A similar structural evolution with the magnetic field was found for other temperatures except for temperatures higher than $22.0^{\circ} \mathrm{C}$, at which the second-level stable hexagonal structure is no longer observed in this experiment. It is predicted that the second-level stable hexagonal structure will appear for $H$ higher than $50.13 \mathrm{kA} / \mathrm{m}$. In addition, the $d(H)$ curve at a higher temperature is significantly shifted to the up-right region in the $d-H$ configuration, as shown in Fig. 1. This implies that the critical fields become higher as the temperature is raised. Also, a larger column spacing $d$ is obtained at a higher temperature under a given field strength.

At a higher temperature, the kinetic energy of the magnetic particles is increased. This leads to an enhancement in the dispersion of the magnetic particles to the liquid carrier. Thus, a higher magnetic field strength is needed to suppress the effect of the kinetic energy of particles forming columns and to achieve a certain structural state in the magnetic fluid film at a higher temperature. This results in a higher critical field at a higher temperature for the same structural state. On the other hand, under a given field strength, there are less particles able to agglomerate at a higher temperature because the particles at higher temperatures possess 


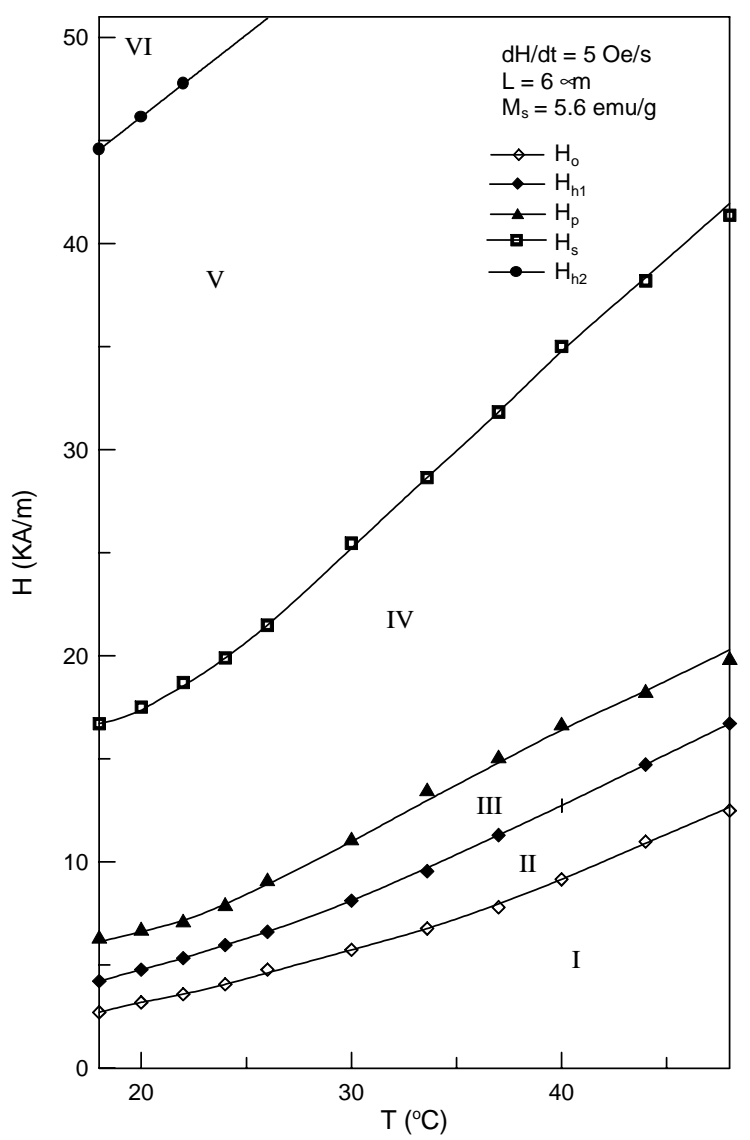

Fig. 2. Phase diagram in the $H-T$ configuration for the structures of the magnetic fluid film: (I) monodispersion; (II) disordered (agglomeration); (III) dynamic hexagonal structure (agglomeration); (IV) first-level stable hexagonal structure; (V) dynamic hexagonal structure (splitting); and (VI) second-level stable hexagonal structure.

higher kinetic energy. Consequently, less magnetic columns can be formed. This causes a larger column spacing $d$ of the ordered structure at a higher temperature for a given field strength, as observed for the $d(H)$ curves shown in Fig. 1.

To illustrate the relationship between the effects of temperature $T$ and of the magnetic field $H$ on the structure formation in the magnetic fluid film, a phase diagram is constructed for the structure pattern in the $H-T$ configuration, as shown in Fig. 2. It is found that the critical fields increase almost linearly with the temperature, but with different slopes for various critical fields. This implies that the effects of temperature and magnetic field on the structural formation in the magnetic fluid film are in the same order. In Fig. 2, the variation in the critical field $\mathrm{H}_{0}$, corresponding to the phase transition or the agglomeration of magnetic particles of the magnetic fluid film under an external magnetic field is much more sensitive than that reported in Ref. [2]. Besides, not only the phase transition from the monodispersion to agglomeration of particles was observed, but also another phase transition at which columns split was found. The corresponding critical field $H_{\mathrm{s}}$ also highly depends on the temperature. It is noted in Fig. 2 that the variation in $d$ at the dynamic hexagonal structure under $H_{\mathrm{h} 1}<H<H_{\mathrm{p}}$ is due to the formation of new columns agglomerated by particles, whereas the formation of another dynamic hexagonal structure under $H_{\mathrm{s}}<H<H_{\mathrm{h} 2}$ is caused by the split of columns.

\section{Conclusion}

The dispersion of magnetic particles is enhanced at higher temperatures due to the increase in kinetic energy. This contributes to higher critical fields in order to compensate for the effect of temperature on the structure formation in the magnetic fluid film under perpendicular magnetic fields.

\section{References}

[1] F.H. Charles, J. Colloid Interface Sci. 52 (1975) 239.

[2] R.E. Rosensweig, J. Popplewell, Int. J. Appl. Electromagn. Mater. 2 (suppl.) (1992) 83.

[3] S. Taketomi, S. Ogawa, H. Miyajima, S. Chikazumi, IEEE Trans. Magn. 4 (1989) 384.

[4] Hao Wang, Yun Zhu, C. Boyd, Weili Luo, A. Cebers, R.E. Rosensweig, Phys. Rev. Lett. 72 (1994) 1929.

[5] C.-Y. Hong, H.E. Horng, I.J. Jamh, C.J. Hsu, Y.D. Yao, H.C. Yang, J. Appl. Phys. 81 (1997) 4275.

[6] J.-C. Bacri, A. Cebers, R. Perzynski, Phys. Rev. Lett. 72 (1994) 2705.

[7] C. Flament, G. Pacitto, J.-C Bacri, I. Drikis, A. Cebers, Phys. Fluids 10 (1998) 2464.

[8] I.J. Jang, H.E. Horng, J.M. Wu, C.-Y. Hong, Y.C. Chiou, H.C. Yang, J. Magn. Magn. Mater. 201 (1999) 317.

[9] C.-Y. Hong, H.-E. Horng, F.C. Kuo, S.Y. Yang, H.C. Yang, J.M. Wu, Appl. Phys. Lett. 75 (1999) 5904.

[10] C.-Y. Hong, C.H. Ho, H.E. Horng, C.-H. Chen, S.Y. Yang, Y.P. Chiu, H.C. Yang, Magn. Gidrodin. 35 (1999) 364.

[11] H.E. Horng, S.Y. Yang, C.-H. Chen, Y.P. Chiu, C.A. Chen, C.-Y. Hong, H.C. Yang, J. Magn. Magn. Mater. 226-230 (2001) 1924. 